This is the final accepted manuscript of:

\author{
A NEW INTEGRATED TLC/MU-ATR/SERS ADVANCED APPROACH FOR THE \\ IDENTIFICATION OF TRACE AMOUNTS OF DYES IN MIXTURES \\ Sciutto Giorgia, Prati Silvia, Bonacini Irene, Litti Lucio, Meneghetti Moreno, Mazzeo \\ Rocco \\ Cite this article as: \\ Sciutto, G., Prati, S., Bonacini, I., Litti, L., Meneghetti, M., Mazzeo R. Anal Chim \\ Acta (2017) 991:104 \\ DOI: https://doi.org/10.1016/j.microc.2018.03.007 \\ First Online: 25 August 2017 \\ Available at: \\ https://www.sciencedirect.com/science/article/pii/S0003267017309546?via\%3Dihub \\ (c) 2017 Elsevier. All right reserved
}




\title{
A new integrated TLC/MU-ATR/SERS advanced approach for the identification of trace amounts of dyes in mixtures
}

\author{
Giorgia Sciutto ${ }^{\text {a, }{ }^{* *}, \text { Silvia Prati }}{ }^{\text {a, }}{ }^{*}$, Irene Bonacini ${ }^{a}$, Lucio Litti ${ }^{b}$, Moreno Meneghetti ${ }^{b}$, \\ Rocco Mazzeo ${ }^{a}$ \\ ${ }^{a}$ Microchemistry and Microscopy Art Diagnostic Laboratory, Department of Chemistry, University of Bologna, Via Guaccimannni 42, 48121, Ravenna, Italy \\ ${ }^{\mathrm{b}}$ Department of Chemical Science, University of Padova, via Marzolo 1, 35131, Padova, Italy
}

\section{H I G H L I G H T S}

- An innovative TLC method coupled to enhanced vibrational techniques was developed.

- SERS and MU-ATR may be performed on the separated spots.

- New AgI@Au TLC plates are proposed.

- Enhanced spectra are obtained without the addition of a colloidal solution.

- The new analytical set up is promising for trace analyses.

\section{Keywords:}

SERS

MU/ATR

Enhanced vibrational techniques Dyes

\section{G R A P H I C A L A B S T R A C T}

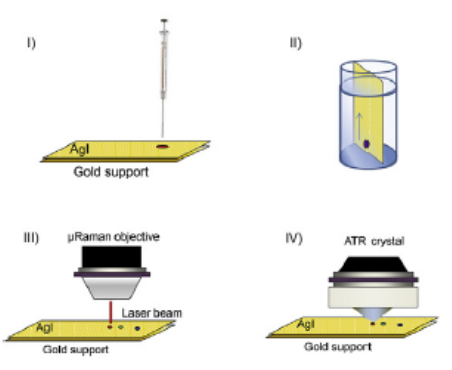

A B S T R A C T

The present research is focused on the setting up of an advanced analytical system for the detection of synthetic dyes. The system is based on the combination of an innovative thin layer chromatography (TLC) plate coupled with enhanced infrared (MU-ATR, metal underlayer attenuated total reflection) and Surface Enhanced Raman (SERS) spectroscopy. In particular, a TLC plate made of silver iodide (AgI) applied onto a gold coated glass slide (AgI@Au) is proposed as an efficient stationary phase for the separation of dyes mixtures. The separated dyes are then identified by means of both enhanced FTIR and SERS, performed directly on the same eluted spots. The use of a mid-IR transparent inorganic salt as stationary phase coupled with the underneath gold layer avoids spectral interferences, enhancing the signal obtained from ATR analyses. At the same time, SERS spectra can be recorded as the TLC plate may act as a SERS active substrate due to the photoreduction of AgI to metallic Ag caused by the exposure to the laser during the Raman analysis. Different mixtures of synthetic dyes of known composition, widely used in dyeing processes, have been tested and the method resulted to be effective in identifying trace amounts in the order of tens nanograms. Moreover, the method has been further evaluated on a real case study represented by dyes extracted from dyed wool.

(ㅇ) 2017 Elsevier B.V. All rights reserved.

\footnotetext{
* Corresponding author.

** Corresponding author.

E-mail addresses: giorgia.sciutto@unibo.it (G. Sciutto), s.prati@unibo.it(S. Prati).
}

\section{Introduction}

Despite the remarkable progresses achieved in the last decades by analytical studies, the characterization of organic dyes still represents a challenging task. Due to their high tinting strength, 
dyes are present in very low concentrations and are usually embedded into multicomponent matrices (such as: paints, textiles, or inks). Nevertheless, their unambiguous and sensitive identification is of outmost importance in forensic studies, food quality controls as well as provenance, dating and authentication studies of art samples [1,2].

When dyes mixtures have to be analyzed, high performance liquid chromatography (HPLC) is probably the analytical technique that provides more information [3,4]. Recently, the sample amount needed for analysis has been largely reduced thanks to the development of advanced methods based on the use of ultra performance liquid chromatography systems coupled with mass-mass (MS/MS) detection [5] or to LC/LC set ups [6].

Nevertheless, the analysis of dyes by means of spectroscopic techniques may complement the above mentioned analyses, as they are versatile in identifying both the organic and the inorganic materials constituting multicomponent samples and are widely available in many conservation/restoration laboratories.

Surface Enhanced Raman Scattering (SERS) microscopy has become increasingly popular and it is, nowadays, a powerful and well established technique for the sensitive molecular detection and identification of dyes on very limited amount of samples $(0.1-0.5 \mathrm{mg})$ [7-9] as well as by means of in-situ set ups [10-12]. On the other hand, infrared microscopy (FTIR) has been sporadically applied to the identification of dyes on dyed samples. In fact, interferences arising from the sample matrices, which may totally hinder the absorption bands of the colorant [13] represent a great analytical limitation. The use of enhanced FTIR methods [13,14], allowing the detection of dyes in micro-extracts, have been proposed in order to overcome this limitation. In particular, Metal Underlayer ATR spectroscopy (MU-ATR) has been proposed for the analyses of few nano-grams of dyes spotted on a gold-coated glass slide [14]. In fact, it has been shown that when the analyte is applied on a gold-coated glass slide and has a thickness larger than monolayers but thinner than the wavelength (i.e. in the order of hundred nanometers), enhanced dyes' spectra can be recorded [14].

When single dyes present in a mixture have to be analyzed, the integrated use of thin layer chromatography (TLC) and SERS has been proposed. In particular, SERS analyses can be performed onto the eluted TLC spots by adding an aliquot of a colloidal solution. This method has been widely applied for instance to the environmental [15], food [16], medical [17] and conservation fields [18-20].

However, the routinely application of this method has some drawbacks due to the partial solubilization that the applied colloidal solution can cause on dye spots located in adjacent positions. In order to overcome this limitation, Ag inkjet-printed SERS papers [21] and silver nanorod arrays fabricated by oblique angle deposition on glass [22] have been proposed.

It is worth mentioning that because of the strong infrared absorption signals of both conventional TLC silica plates and SERS active substrates, FTIR analyses cannot be performed on these type of plates [23]. Therefore, TLC/FTIR set ups have been usually performed by using the transfer approach [24,25], a time consuming procedure which exposes the analytes to the risk of sample loss and contamination. In an attempt to avoid drawbacks related to the strong background absorptions in the mid-IR range (400$4000 \mathrm{~cm}^{-1}$ ) shown by the traditional TLC stationary phases (such as silica and alumina), AgI has been recently proposed for the setup of narrowband TLC plates [26,27].

The aim of this research is to propose for the first time a new TLC plate that allows the analysis of trace amounts of dyes present in mixture by means of the integrated use of enhanced Raman (SERS) and enhanced FTIR spectroscopy (Mu-ATR), obtaining complementary results and overcoming the previous observed limitations in terms of contamination and sensitivity. A thin layer of AgI deposited on a gold coated glass slide (AgI@Au) has been used as stationary phase. AgI has been chosen because, as previously reported [26], it allows the rinse of organic solvents by capillarity and it is transparent in the mid IR. Moreover, SERS spectra can be obtained on the same eluted spots, exploiting the AgI photo reactivity. Indeed, silver halides can absorb the incident light, generating electrons in the conduction band ( $\mathrm{CB}$ ) and holes in the valence band (VB). Then, the photo-generated electrons in the CB are captured by surface lattice $\mathrm{Ag}^{+}$ions to form metallic $\mathrm{Ag}^{\mathrm{n}}$ clusters, whereas photo-generated holes in the VB oxidize lattice anions $\mathrm{X}^{-}$to $\mathrm{X}_{2}$, which is released as a consequence [28]. A photo-induced selfstability mechanism has also been demonstrated for AgX compounds. Irradiated AgX nanoparticles are transformed into a stable $\mathrm{Ag} / \mathrm{AgX}$ hybrid nanocomposite after early in situ formation of surface $\mathrm{Ag}^{0}$ [29-31]. Significantly, hybrid Ag/AgX-based nanomaterials proved to be able to support localized surface plasmons upon exposure to visible light [28,32-36]. Concerning the elucidation of the enhancement mechanism, previous researches carried out on different silver halides derivatives underlined that laser irradiation produces photolytic silver particles, which play a key role in the generation of SERS effects [34,35]. On the other hand, SERS amplification is also promoted by the formation of complexes with the $\mathrm{Ag}+$ ions formed on the silver halides nanoparticles [33].

The novel TLC based analytical protocol illustrated in this paper presents several advantages with respect to already published methods. In particular, the integrated use of two complementary enhanced techniques can be applied after the separation of mixtures of analytes, without the addition of any colloidal solution. In this way, the complementary information obtained by MU-ATR and SERS analyses may allow to better characterize the mixture's components, achieving very low detection limits thanks to the spectral enhancement.

Performances of the new TLC active plates were tested for the characterization of different mixtures of synthetic dyes and validated on a micro extract obtained on less than $0,01 \mathrm{mg}$ of wool, died with a mixture of dyes. The choice of such analytes is strictly related to the important role they have been playing in industrial applications and in the forensic field $[9,37]$. Actually, since the half of 19 th century, they have been widely used in dyeing processes.

\section{Experimental section}

\subsection{Materials}

Silver nitrate $\left(\mathrm{AgNO}_{3}\right)$, potassium iodide $(\mathrm{KI})$, trisodium citrate dihydrate and all the analyzed dyes (see Table 1) were purchased from Sigma Aldrich. Gold coated glass slides (Au $200 \mathrm{~nm}$ thick) were acquired from Thermo Fisher Scientific. AgI particles were filtered using Whatman filter papers with a diameter of $125 \mathrm{~mm}$ and a particle retention $<2 \mu \mathrm{m}$. HPTLC were performed using nanosilica plates purchased by Fluka with layer thickness of $0.2 \mathrm{~mm}$ and medium pore diameter of $60 \AA$. The citrate silver colloid paste was prepared following the standard procedure for the characterization of organic dyes in historical samples $[18,38]$.

\subsection{Instrumentation}

FTIR ATR analyses were performed by using a Thermo Nicolet iNTM10MX microscope, fitted with an MCT detector cooled by liquid nitrogen. Spectra were recorded in the range $4000-675 \mathrm{~cm}^{-1}$ with an optical aperture of $200 \times 200 \mu \mathrm{m}$, using a spectral resolution of $4 \mathrm{~cm}^{-1}$ and 128 scans. Raman spectra were collected with a Bruker Santerra microscope equipped with a charge coupled device (CCD) detector and using an excitation source emitting at $785 \mathrm{~nm}$, with a 
Table 1

Synthetic dyes submitted to the spectroscopic analysis.

\begin{tabular}{llll}
\hline Name & C.I. index & Code & Dye class \\
\hline Diamond Green & 42040 & Triarylmethane
\end{tabular}

Methyl violet

42535

Crystal violet

Rhodamine B

Rhodamine 6G

Eosin A

Martius yellow

Cochineal red A

Fast red AV
42555

45170

45160

45380

10315

16255

15620
BV1

Triarylmethane

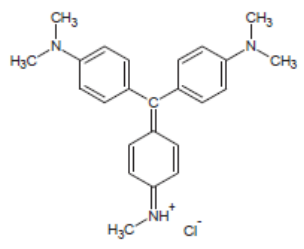

BV3

Triarylmethane

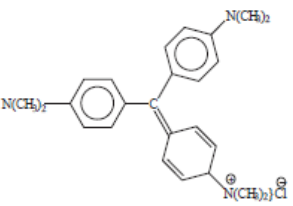

Xanthene

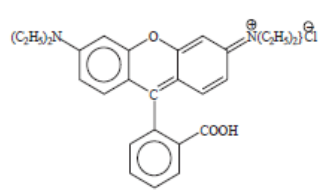

Xanthene

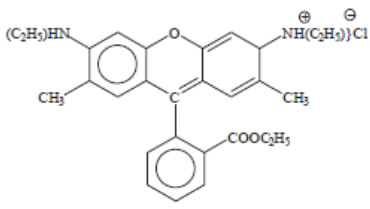

AR87

Xanthene<smiles></smiles>

Nitro<smiles>O=[N+]([O-])c1cc([N+](=O)[O-])c2ccccc2c1O</smiles>

AR18

Azo

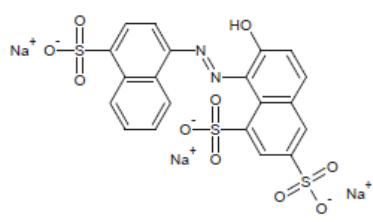

Azo

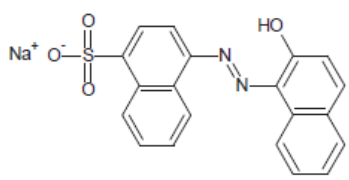


Table 1 (continued)

\begin{tabular}{llll}
\hline Name & C.I. index & Code & Dye class \\
\hline Indigo carmine, & 73015 & Indigoid
\end{tabular}

power of about $10 \mathrm{~mW}$. All the spectra were acquired with a single integration time of $3 \mathrm{~s}$, focusing on the dyes dried spots with an Olympus $20 \times$ microscope objective. The scanning electron microscopy (SEM) images were obtained with a Quanta Inspect S FEI instrument operating at $25 \mathrm{keV}$ with a tungsten filament current $100 \mu \mathrm{A}$ for the acquisition of the secondary electrons (SE) images. The instrument was equipped with an Edax Phillips New XL30 spectrometer. Energy dispersive XRay microanalysis was performed at $25 \mathrm{keV}$ with $100 \mathrm{~s}$ of lifetime, within an area of $25 \times 25 \mu \mathrm{m}$. Xray powder diffraction patterns were recorded on a PanAnalytical X'Pert Pro equipped with an X'Celetrator detector powder diffractometer using $\mathrm{Cu}$ Ka radiation generated at $40 \mathrm{kV}$ and $40 \mathrm{~mA}$. The diffraction patterns were collected within the $2 \theta$ range from $20^{\circ}$ to $60^{\circ}$ with a step size $(\Delta 2 \theta)$ of $0.05^{\circ}$ and a counting time of $150 \mathrm{~s}$.

AFM images were obtained on the AgI layer applied on gold, by using a Digital NanoScope IIIa Multimode (Veeco, USA) operating in tapping mode with $(\mathrm{n})$ doped Si probes (spring constant, 20-80 $\mathrm{Nm}-1$; resonance frequency, $200-400 \mathrm{kHz}$; nominal tip radius, < $10 \mathrm{~nm})$.

\subsection{AgI synthesis and deposition method}

$0.1 \mathrm{M}$ aqueous solution of $\mathrm{AgNO}_{3}$ and $\mathrm{KI}$ were cooled at $4{ }^{\circ} \mathrm{C}$ for 30 minThen $\mathrm{AgNO}_{3}$ solution was added to the KI solution and the mixture was kept at $4{ }^{\circ} \mathrm{C}$ for $1 \mathrm{~h}$. Subsequently the obtained yellowish solution was maintained at room temperature for $50 \mathrm{~min}$. The filtration was performed under vacuum created by a water pump and the precipitate was rinsed with ultrapure water to remove precursor reagents. The powder was subsequently dried in an oven at $40{ }^{\circ} \mathrm{C}$ for $15 \mathrm{~min}$. Afterwards, $0.100 \mathrm{gr}$ of AgI was suspended in $7 \mathrm{ml}$ of isopropanol and sonicated for $30 \mathrm{~min}$ in an ultrasonic bath. Then, $1 \mathrm{ml}$ of the obtained surnatant suspension was applied on a gold slide by drop casting deposition and dried in an isopropanol saturated chamber for $24 \mathrm{~h}$. All the preparation steps as well as the experiments were carried out in the dark.

\subsection{TLC analysis}

Dyes solutions and mixtures $(0.5 \mu \mathrm{L}$ at the concentration of $10^{-4} \mathrm{M}$ ) were spotted on the AgI@Au TLC plates near to the edge of the substrate by using a Hamilton syringe. Chromatographic separation was performed using as eluent a mixture of ethanol and ammonia $9: 1 \mathrm{v} / \mathrm{v}$.

\subsection{Wool dyeing and extraction method}

An historical recipe reported in the sample book developed by Adolf Lehne in 1893 [39] was followed for the dying process. Briefly, $1 \mathrm{mg}$ of raw clean wool was immersed for $30 \mathrm{~min}$ in the dyeing bath prepared with $5 \mathrm{ml}$ solution of BG1, BB9 and BV10 in ultrapure water (with a final concentration of $10^{-4} \mathrm{M}$ each) and warmed up to $70{ }^{\circ} \mathrm{C}$. Then, after a slow cooling down at room temperature, dyed fibers were rinsed with ultrapure water to remove any unbound dye component and dried in oven at $40{ }^{\circ} \mathrm{C}$ for $20 \mathrm{~min}$.

The extraction procedure was performed with methanol on less than $0,01 \mathrm{mg}$ of the dyed wool (corresponding to few $\mathrm{mm}$ in length of a single fiber) following an ad hoc developed procedure for analysis of synthetic dyes [13]. Subsequently, $0.5 \mu \mathrm{L}$ of the extracted solution was applied on AgI@Au TLC plate for the chromatographic analysis.

\section{Results and discussion}

\subsection{Characterization of the AgI@Au TLC plates}

Synthesis and deposition of the AgI were set up in an attempt to optimise the film homogeneity, the chromatographic separation and the signal enhancement. Moreover, the synthesis was performed without the addition of stabilizers [40], which could be responsible of spurious signals in the IR and Raman spectra.

The crystalline properties of the synthetized AgI powder were characterized by XRD analyses (Fig. SI 1). Results showed the presence of $\gamma$ AgI cubic crystal phase (JCPDS Card No: 090399) characterized by the presence of Bragg diffraction peaks centered at $2 \theta=23.7,39.1$ and 46.3 that corresponded to (111), (220) and (311) planes, respectively. Moreover, diffraction peaks at $2 \theta=22.3$ and 25.3 , ascribable to the $\beta$ AgI hexagonal phase and corresponding to (100) and (101) planes (JCPDS Card No: 01-078-1614) were also identified. All these features are consistent with scientific literature [41,42].

UV/Vis spectra of AgI suspensions collected from the synthetized powder showed characteristic features in the visible and near UV spectral region. The absorption edge is extended to about $450 \mathrm{~nm}$ (Fig. SI 2), which is consistent with what is reported in literature [43] and corresponds to the direct band gap excitation (W1,2), responsible for the yellow color of the material.

The deposition of AgI on gold coated glass slides was performed by drop casting, after prolonged sonication of an AgI suspension in isopropanol. Afterwards, the Agl@Au system was kept in an isopropanol saturated chamber for $24 \mathrm{~h}$, to assure a slow and uniform evaporation of the solvent. Morphology and microstructure of AgI thin films were investigated by SEM and AFM. Secondary electron (SE) images showed that irregular spheroidal aggregates of AgI were present with different sizes (Fig. 1a) ranging from 100 to $900 \mathrm{~nm}$. Statistically, the most frequent AgI aggregates size is centered between 200 and $300 \mathrm{~nm}$ (Fig. 1b).

By means of AFM it has been possible to observe an average particle diameter ranging from $6.5 \mathrm{~nm}$ to $24 \mathrm{~nm}$ (Fig. 1c). In addition, AFM analyses allowed also estimating the thickness of the AgI layer, which resulted to have an average value of $2 \mu \mathrm{m}$. 
a)
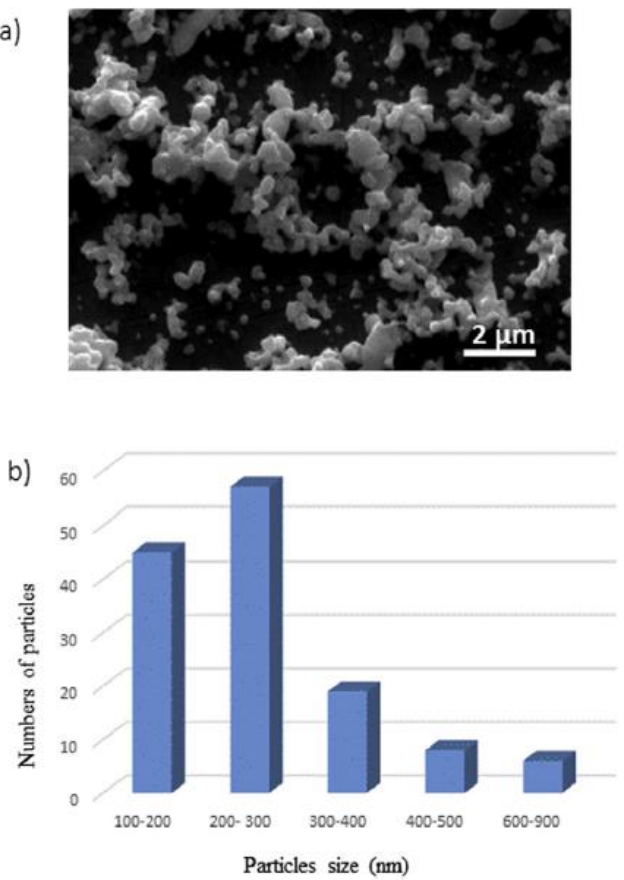

c)

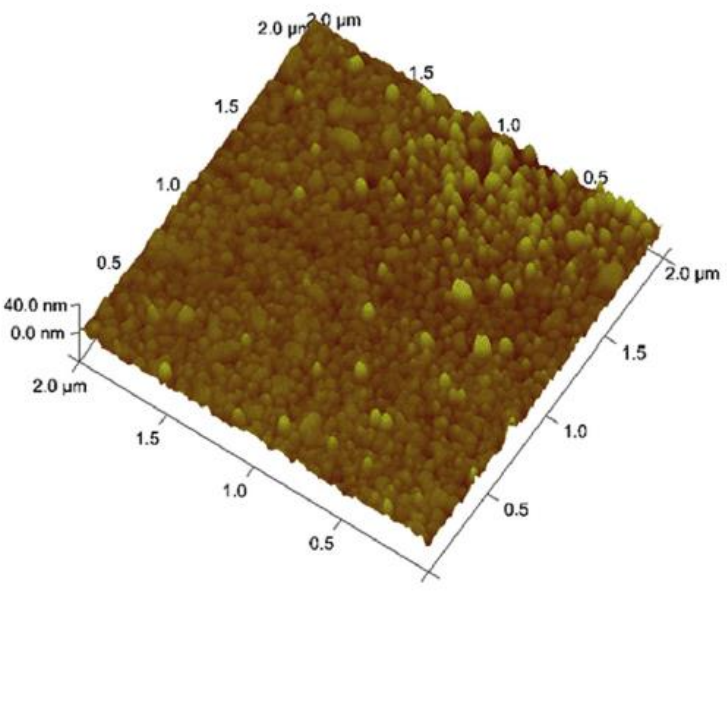

Fig. 1. a) SE image of AgI particles deposed on an Au substrate; b) Particles size distribution histogram; c) AFM image of AgI particles deposed on an Au substrate.

\subsection{Enhanced FTIR and Raman analyses on AgI@Au}

A preliminary study was performed to evaluate the possibility to use AgI@Au as a MU-ATR and SERS active substrate on a selection of 11 basic and acid dyes.

In an attempt to verify the possible influence of AgI on intensity, shape and position of the absorption bands, analyses were carried on both AgI coated and uncoated gold substrates. After the deposition of $0.5 \mu \mathrm{L} 10^{-4} \mathrm{M}$ of the dye solutions on the gold coated glass slides a differential evaporation rate occurs across the drop [44], which is responsible of the accumulation of the dye along the drop borders (the so called "coffee ring" effects). Such effect was less notable on the Agl@Au plates, ensuring a more homogeneous distribution of the dye (Fig. 2b). Therefore, a key role played by AgI in the water evaporation rate can be inferred.

ATR spectra recorded on both AgI@Au and Au plates present similar features, with just some slight differences in the relative intensity of the bands (see Fig. 2a BG1 spectra as example and SI Table 1 , which reports a tentative assignment of absorption bands for all the investigated dyes).

In order to estimate the influence of AgI in the enhancement of the FTIR spectra, the average peak areas of some characteristic bands of BG1 have been calculated and reported in Fig. 2c. It can be noted that absorption signals are of the same order of magnitude, even if spectra recorded on AgI are slightly less intense. This evidence can be related with the distribution of the dye, which is not concentrated on the ring of the drop, but homogeneously distributed in the circular area formed after solvent evaporation. Nevertheless, the observed slight signal reduction is acceptable and it can be said that MU-ATR enhancement of spectra can be obtained also by using Ag@@Au plates thanks to the transparency of AgI and the limited thickness of the deposition. In a previous paper we showed that the enhancement effect observed in MU-ATR was 20-80 times greater than that achievable with transflectance and allowed to reach a limit of dye detection of few ng [14].
It is known from scientific literature that $\mathrm{AgI}$ has been used as a SERS provider [36]. To verify its effectiveness, all the investigated dyes were analyzed on the AgI@Au plates (SI Table 2 reports the spectra of all the analyzed dyes as well as a tentative bands assignment).

Fig. 3 reports the Raman spectra collected from a $0.5 \mu \mathrm{L}$ solution of BG1 $10^{-4} \mathrm{M}$ spotted on different supports (AgI@Au, AgI@SiO $\mathrm{Au}$ ). Sensible enhancement of the vibrational features are achieved analysing the dye spotted on the AgI@Au plates (Fig. 3a). The calculation of the enhancement factor represents a challenging task when solid substrates are used as SERS providers. In an attempt to provide a tentative evaluation of the enhancement effects, the average peak areas of some specific BG1 bands have been measured and reported in Fig. 3b. The average enhancement factors (intended as ratio of the average peaks areas) are in the order of 150-300 with a preferential enhancement observed for low wavenumber spectral bands. When SERS analyses are performed, a detection limit is hard to define. Indeed the signal amplification depends on the contact between the analyte and the SERS substrate, which may even improve decreasing the analyte concentration. However, the experimental data showed that when 20 ng of BG1are spotted on an Au support very noisy spectra are recorded while, when the same amount is spotted on Agl@Au, the dye can be easily identified. To compare the method with procedures already published, SERS spectra were recorded analysing the same amount of dye spotted on a HPTLC nano silica plate (the same plates will be used as comparison in par. 3.3.1) after the addition of a silver colloidal paste prepared as reported in Ref. [18]. Differently from what obtained on glass slides, the addition of the colloidal paste allowed identifying weak features of BG1 (Fig. 3c). However, a clear identification can be obtained when $200 \mathrm{ng}$ of BG1 were analyzed at the presence of the Ag colloidal paste (Fig. 3c), but the resulting signals were weaker than what obtained on 20 ng of BG1 spotted on AgI@Au, thus confirming that the new support is promising if compared to already published systems. 


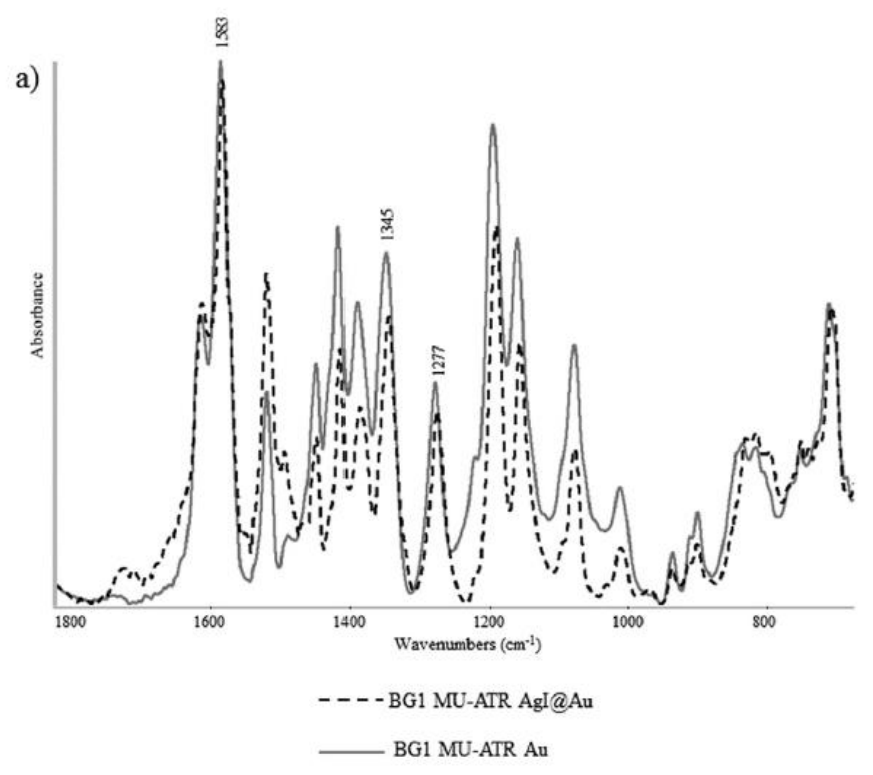

b)
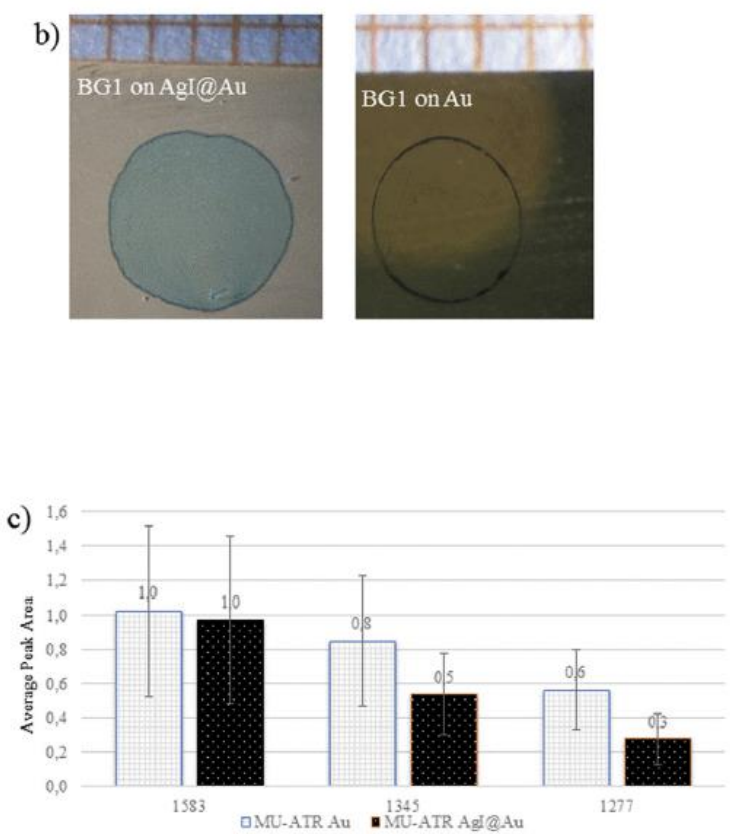

Fig. 2. a) MU-ATR spectra of BG1 $10^{-4}$ M solution acquired on an Agl@Au plate and on a gold coated glass slide; b) BG1 applied on an Agl@Au plate and on a gold coated glass slide (Au) after solvent evaporation; c) Average peak areas of some characteristic IR bands of BG1 $10^{-4} \mathrm{M}$ solution acquired on an Agl@Au plate and on a gold coated glass slides.
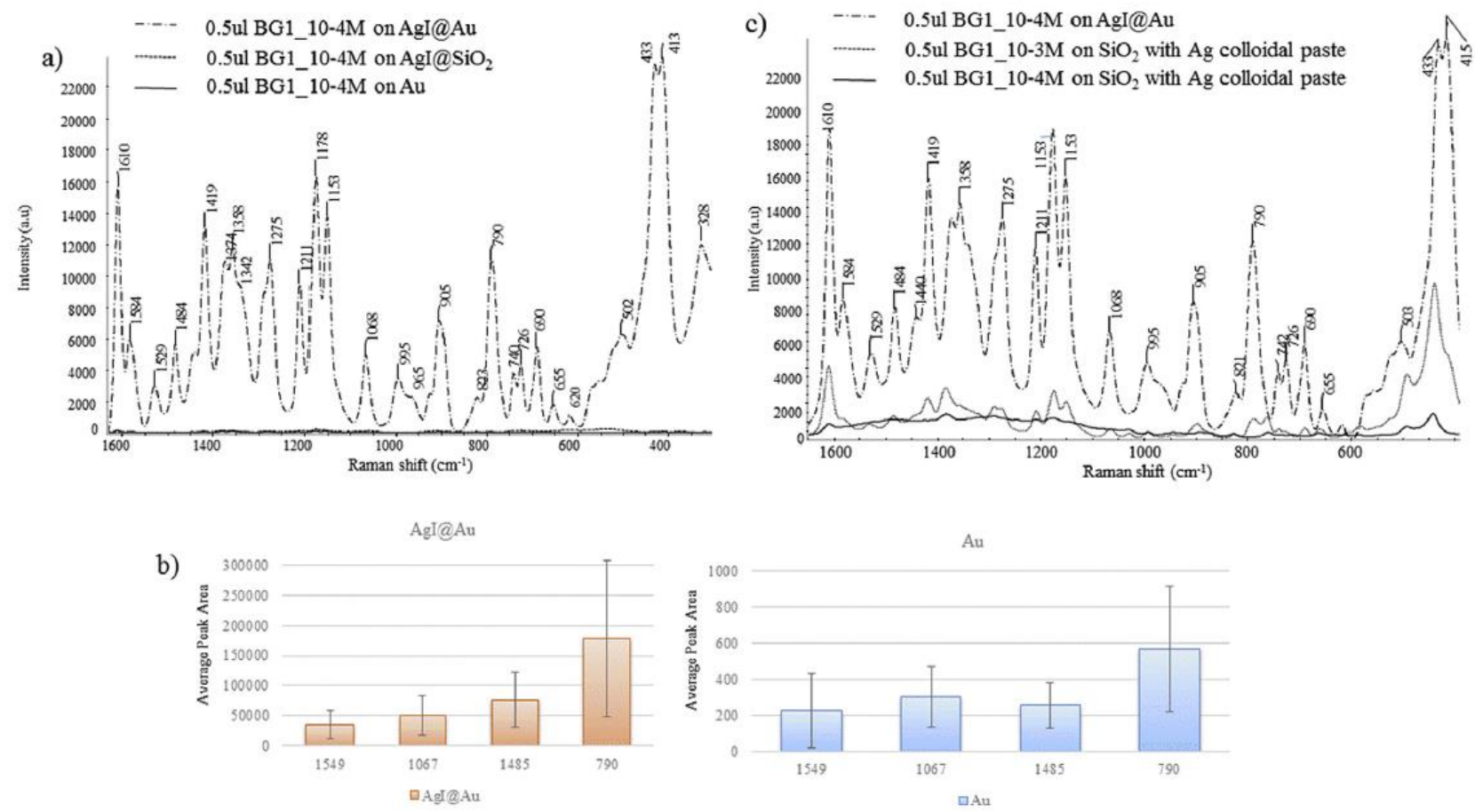

Fig. 3. a) Raman spectra of BG1 $10^{-4} \mathrm{M}$ solution acquired on $\mathrm{AgI@Au,} \mathrm{AgI@SiO}$ 2 and Au b) Average peak areas of some characteristic Raman bands of BG1 $10^{-4} \mathrm{M}$ solution acquired on an Agl@Au plate and on a gold coated glass slide c) Raman spectra of BG1 $10^{-4} \mathrm{M}$ solution acquired on Agl@Au compared to SERS spectra of BG1 $10^{-4} \mathrm{M}$ and $10^{-3} \mathrm{M}$ solutions acquired on HPTLC nano silica plates after the addition of a silver colloidal paste.

In order to verify if the SERS effect was a result of the local field enhancement of the photolytic silver particles $\left(\mathrm{Ag}^{0}\right)$ produced in situ by the laser beam $[33,34,45]$, SEM observations were systematically carried out on the Agl@Au plates before and after the laser treatment. Interestingly, it was evident that the laser irradiation of the AgI@Au plates converted the originally smooth surfaces 
of aggregates in nano-rough substrates (Fig. 4). Moreover, the nanoparticles produced after laser irradiation had the same morphology of nanoparticles identified as silver nanoparticles (AgNPs) obtained by laser treatment of silver salts in already published papers [34,46-48].

To better elucidate the possible role played by the gold underlayer in spectral enhancement, Raman spectra of dyes were recorded an AgI film deposited directly on glass slides $\left(\mathrm{AgI} @ \mathrm{SiO}_{2}\right)$. Interestingly, in this case by applying the same experimental conditions used for the analyses on AgI@Au substrates it has not been possible to detect the same amplified signal (Fig. 3a). SEM morphological observations of $\mathrm{AgI@SiO} 2$ showed that the creation of new small particles on the surface was still detectable after the laser treatment, but the amount of them and the general changes in cluster morphology appeared considerably more limited (SI Fig. 3) if compared with the effect obtained on AgI@Au substrates. These outcomes highlights the crucial role played by the gold support in the photoreduction of AgI as well as in the electromagnetic properties of the system.

\subsection{TLC separation coupled to enhanced FTIR and Raman analyses on AgI@Au}

\subsubsection{Set up of the TLC/MU-ATR/SERS integrated analytical method} on standard solutions

TLC tests have been performed on a selection of dyes. The first solution (dye mixture A), characterized by a bright violet tonality, contained three different basic dyes (BV10, BG1 and BB9). Fig. 5a reports the separation obtained from $0.5 \mu \mathrm{L}$ of mixture $\mathrm{A}$ (with a final concentration of $10^{-4} \mathrm{M}$ for each dye) with an eluent composed by ethanol (EtOH) and ammonium hydroxyde $\left(\mathrm{NH}_{3}\right)$ 9:1 $\mathrm{v} / \mathrm{v}$. The eluent allowed the separation of the three dye components without any overlapping among them. Moreover, the short distance ran by the eluent front allowed a limited dilution and diffusion of the dyes on the TLC plate, assuring a higher intensity of the spectroscopic related signals (MU-ATR and SERS spectra are reported in SI Fig. 4).

The same eluent was tested on mixture B (Fig. 5b) containing acid and basic dyes [AR87 (xanthene dye) AR18 (azo dye) and BV1 (triarylmethane dye)]. Analogous good performances in terms of selectivity have been achieved with this chromatographic system

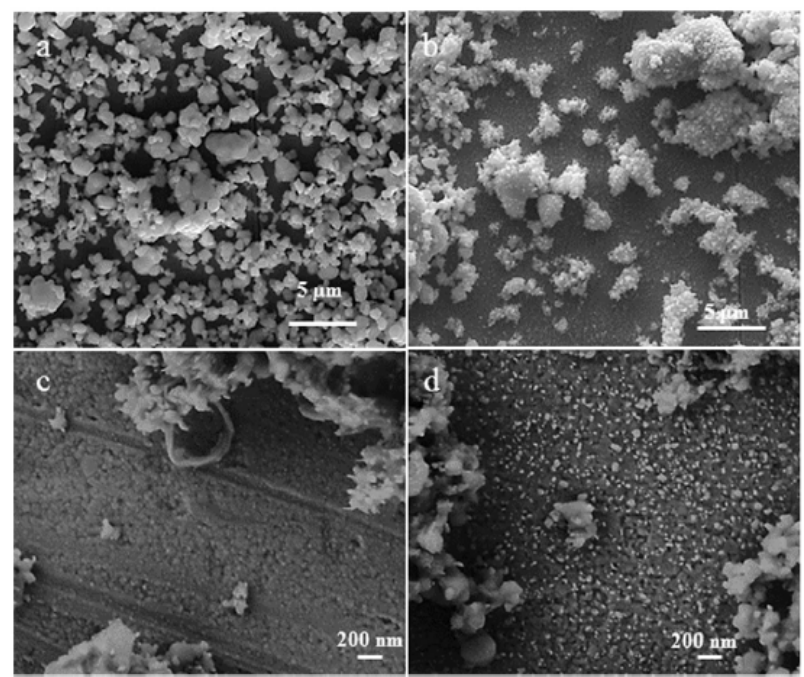

Fig. 4. SEM images of Ag@@Au substrate before (a, c) and after (b, d) laser irradiation. a)

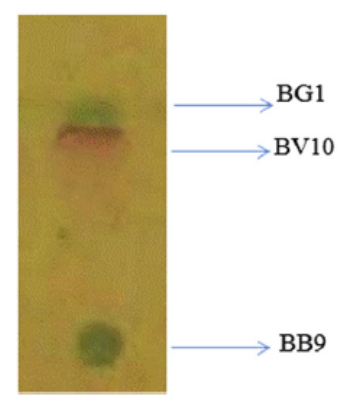

b)

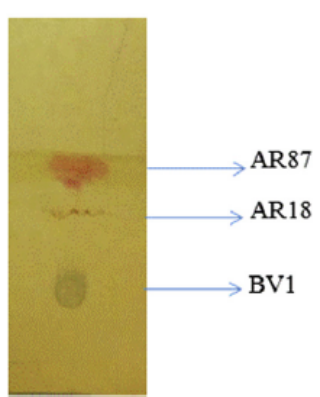

Fig. 5. a) Dye mixture A and b) Dye mixture B after TLC development on Ag@@Au plates.

\section{(Fig. 5b).}

All six analytes have been identified by their spectral fingerprints, which were still well recognizable in both the enhanced Raman and FTIR spectra (SI Figure 4 and SI Table 1).

Mixture A was analyzed by SERS after the separation on HPTLC nano silica plates to compare the AgI@Au method with a TLC-SERS method already reported in literature [7]. To this purpose, few microliters of citrate reduced silver colloidal paste were applied on the eluted dye spots in order to ensure the collection of the SERS signals. Difficulties were incurred both in the identification of the proper area within the dye spot (due to the hampering effect of the colloid) as well as in the collection of data. Indeed, to assure the acquisition of an enhanced spectrum, Raman spectra should be collected before the complete drying of the colloidal paste. Moreover, the deposition of the colloidal paste on the eluted spots may lead to contamination effects as well as may affect results reproducibility. Because of all these drawbacks, only the dye BB9 (data not shown) was identified on the HPTLC plates. In addition, it has not been possible to analyse the spots eluted on nano silica HPTLC plates by means of FTIR microscopy.

\subsubsection{Validation of the TLC/MU-ATR/SERS integrated analytical method on micro extracts from died wool}

The validation of the advanced AgI@Au TLC substrate was performed on dyes extracted from dyed wool fibers. This methods can be of great interest when low amount of sample is available, as it occurs in forensic science and cultural heritage materials. A few fibers of wool, dyed with the mixture A, were submitted to an ad hoc mild extraction method based on the use of less than $0.01 \mathrm{mg}$ of sample [13]. The extracted solution was applied on the AgI@Au TLC plate and the dyes eluted. Raman and FTIR enhanced spectroscopic measurements were then performed directly onto the three eluted spots. Both techniques succeeded in the identification of the dye mixture components as it can be seen by comparing spectra reported in Fig. 6 with the reference spectra reported in SI figure 4 and in tables SI-1and SI-2. MU-ATR spectra acquired above the BB9 spots (Fig. SI 5) showed the typical absorption bands at 1578 and $1540 \mathrm{~cm}^{-1}$ of carboxylates. The presence of these compounds is justified because, before dying, the virgin wool was subjected to a washing treatment carried out at $40^{\circ} \mathrm{C}$ by means of Marseille soap. Therefore, it can be said that the integrated use of TLC and enhanced vibrational techniques allowed both the identification of all the three dyes and additional information about the overall dying procedure.

\section{Conclusions}

The present research deals with the setting up of a new 
a) RAMAN SPECTRA
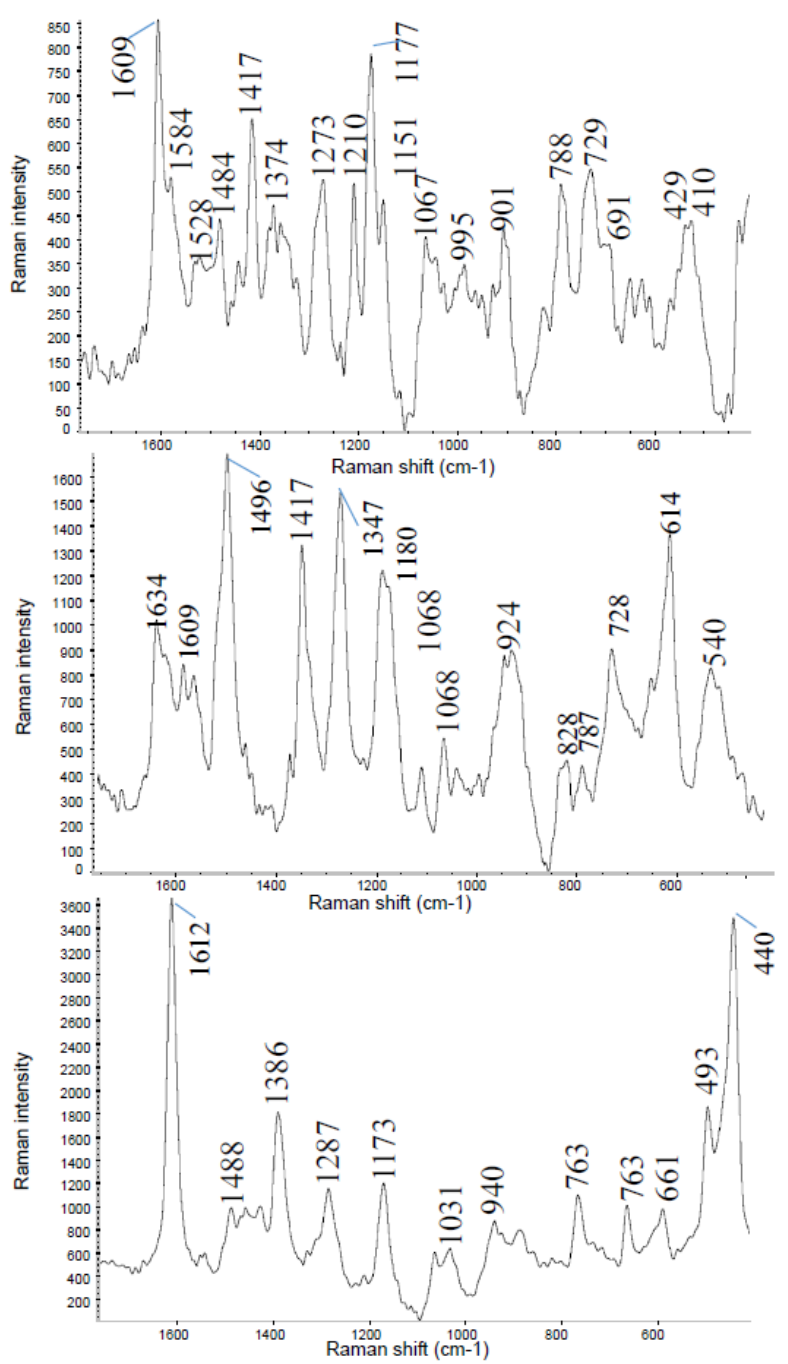

b) FTIR SPECTRA

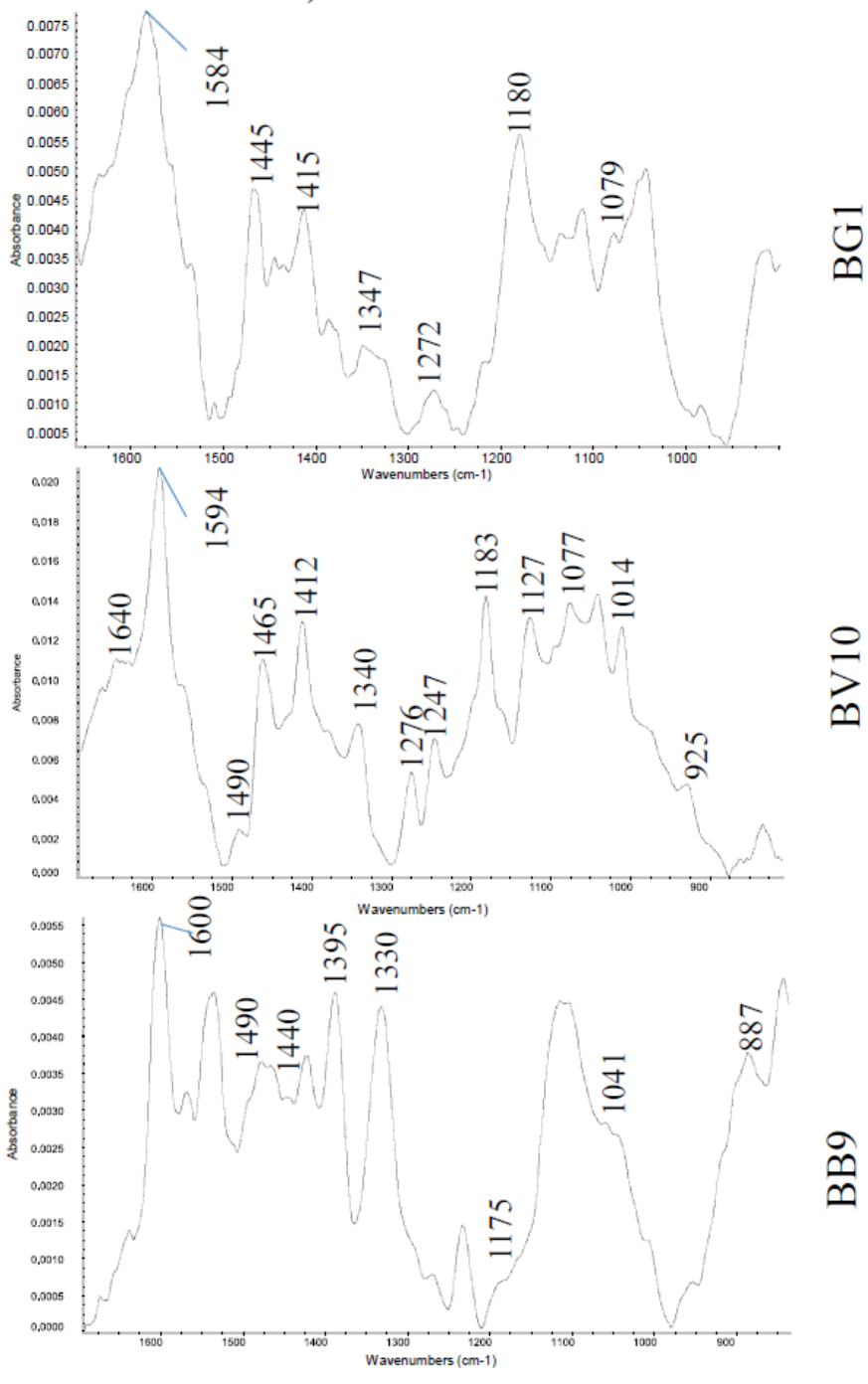

Fig. 6. a) SERS and b) MU-ATR spectra recorded on dyes spots after TLC development of the extracted sample on an Agl@Au plate.

analytical method that couples the chromatographic separation of dyes mixtures by means of a newly developed Agl@Au TLC plate and the chemical characterization of the eluted dye spots with enhanced spectroscopic techniques (MU-ATR and SERS). Trace amount of dyes can be analyzed with this method, thus exploiting the complementary information obtained from Raman and infrared enhanced spectra. In particular, MU-ATR spectra can be recorded directly on the eluted dye spots, thanks to the transparency of AgI in the mid infrared region and the presence of the reflective gold underlayer. AgI@Au plates can also be used as SERS active substrate thanks to the photoreduction properties of the silver salt, which is observed only when AgI is deposited on gold glass slides. Thus, AgI@Au plates allowed to obtain SERS spectra without the addition of a colloidal solution but just exploiting the laser exposure effect, with the advantage of avoiding contamination between the eluted spots. It is worth mentioning the fact that this new system can compete with the traditional TLC approaches coupled to the already available spectroscopic detection. Finally, this new method can found promising applications in all those scientific fields where trace analysis of dyes is requested, such as in the forensic and conservation science fields.

\section{Acknowledgments}

The present work was carried out with the support of the IPERION CH project (Integrated Platform for the European Research Infrastructure ON Cultural Heritage) funded by the European Commission (H2020-INFRAIA-2014-2015, under Grant No. 654028).

The Authors thank Professor Massimo Marcaccio for the AFM analyses.

\section{Appendix A. Supplementary data}

Supplementary data related to this article can be found at http:// dx.doi.org/10.1016/j.aca.2017.08.020. 


\section{References}

[1] F. Pozzi, J.R. Lombardi, S. Bruni, M. Leona, Sample treatment considerations in the analysis of organic colorants by surface-enhanced raman scattering, Anal. Chem. 84 (2012) 3751-3757.

[2] R.W. Jones, McClelland, Analysis of writing inks on paper using direct analysis in real time mass spectrometry, J.F. Forensic Sci. Int. 231 (2013) 73-81.

[3] D.K. Lang, R. Gill, C. Blacklaws, H.M. Bickley, Characterisation of acid dyes in forensic fibre analysis by high-performance liquid chromatography using narrow-bore columns and diode array detection, J. Chromatogr. A 442 (1988) $187-208$.

[4] M.R. van Bommel, The analysis of dyes with HPLC coupled to photodiode array and fluorescence detection, Dyes Hist. Archaeol. 20 (2005) 30-38.

[5] L.G. Troalen, A.S. Phillips, D.A. Peggie, P.E. Barran, A.N. Hulme, Historical textile dyeing with Genista tinctoria L: a comprehensive study by UPLC-MS/MS analysis, Anal. Methods 6 (22) (2014) 8915-8923.

[6] M.R. van Bommel, I.V. Berghe, A.M. Wallert, R. Boitelle, J. Wouters, Highperformance liquid chromatography and non-destructive three-dimensional fluorescence analysis of early synthetic dyes, J. Chromatogr. A 1157 (1) (2007) 260-272.

[7] F. Casadio, M. Leona, J.R. Lombardi, R. Van Duyne, Identification of organic colorants in fibers, paints, and glazes by surface enhanced Raman spectroscopy, Accounts Chem. Res. 43 (6) (2010) 782-791.

[8] C. Lofrumento, M. Ricci, E. Platania, M. Becucci, E. Castellucci, SERS detection of red organic dyes in Ag-agar gel, J. Raman Spectrosc. 44 (1) (2013) 47-54.

[9] I. Geiman, M. Leona, J.R. Lombardi, Application of raman spectroscopy and surface-enhanced raman scattering to the analysis of synthetic dyes found in ballpoint pen inks, J. forensic Sci. 54 (4) (2009) 947-952.

[10] A. Raza, B. Saha, In situ silver nanoparticles synthesis in agarose film supported on filter paper and its application as highly efficient SERS test stripes, Forensic Sci. Int. 237 (2014) e42-e46.

[11] D. Kurouski, R.P. Van Duyne, In Situ detection and identification of hair dyes using surface-enhanced Raman spectroscopy (SERS), Anal. Chem. 87 (5) (2015) 2901-2906

[12] M.V. Cañamares, J.V. García-Ramos, J.D. Gomez-Varga, C. Domingo, S. SánchezCortés, Ag nanoparticles prepared by laser photoreduction as substrates for in situ surface-enhanced raman scattering analysis of dyes, Langmuir 23 (9) (2007) 5210-5215.

[13] S. Prati, M. Quaranta, G. Sciutto, I. Bonacini, L. Litti, M. Meneghetti, R. Mazzeo, Use of nano gold obtained by laser ablation for SEIRA analyses of colorants, Herit. Sci. 2 (1) (2014) 28.

[14] S. Prati, M. Milosevic, G. Sciutto, I. Bonacini, S.G. Kazarian, R. Mazzeo, Analyses of trace amounts of dyes with a new enhanced sensitivity FTIR spectroscopic technique: MU-ATR (metal underlayer ATR spectroscopy), Anal. Chim. Acta 941 (2016) 67-79.

[15] D.W. Li, W.L. Zhai, Y.T. Li, Y.T. Long, Recent progress in surface enhanced Raman spectroscopy for the detection of environmental pollutants, Microchim. Acta $181(1-2)(2014)$ 23-43.

[16] D. Lv, Y. Cao, Z. Lou, S. Li, X. Chen, Y. Chai, F. Lu, Rapid on-site detection of ephedrine and its analogues used as adulterants in slimming dietary supplements by TLC-SERS, Anal. Bioanal. Chem. 407 (5) (2015) 1313-1325.

[17] A. Lucotti, M. Tommasini, M. Casella, A. Morganti, F. Gramatica, G. Zerbi, TLC-surface enhanced Raman scattering of apomorphine in human plasma, Vib. Spectrosc. 62 (2012) 286-291.

[18] C.L. Brosseau, A. Gambardella, F. Casadio, C.M. Grzywacz, J. Wouters, R.P. Van Duyne, Ad-hoc surface-enhanced Raman spectroscopy methodologies for the detection of artist dyestuffs: thin layer chromatography-surface enhanced Raman spectroscopy and in situ on the fiber analysis, Anal. Chem. 81 (8) (2009) 3056-3062.

[19] M.V. Cañamares, D.A. Reagan, J.R. Lombardi, M. Leona, TLC-SERS of mauve, the first synthetic dye, J. Raman Spectrosc. $45(11-12)(2014)$ 1147-1152.

[20] F. Pozzi, N. Shibayama, M. Leona, J.R. Lombardi, TLC-SERS study of Syrian rue (Peganum harmala) and its main alkaloid constituents, J. Raman Spectrosc. 44 (1) (2013) 102-107.

[21] W.Y. Wei, I.M. White, Chromatographic separation and detection of target analytes from complex samples using inkjet printed SERS substrates, Analyst 138 (13) (2013) 3679-3686.

[22] J. Chen, J. Abell, Y.W. Huang, Y. Zhao, On-chip ultra-thin layer chromatography and surface enhanced Raman spectroscopy, Lab a Chip 12 (17) (2012) 3096-3102.

[23] C.J. Percival, P.R. Griffiths, Direct measurement of the infrared spectra of compounds separated by thin-layer chromatography, Anal. Chem. 47 (1) (1975) 154-156.
[24] S.G. Bush, A.J. Breaux, The application of diffuse and specular reflectance to TLC-FTIR and other microsampling applications, Microchim. Acta 94 (1-6) (1988) $17-20$.

[25] G. Glauninger, KA. Kovar, V. Hoffmann, Possibilities and limits of an on-line coupling of thin-layer chromatography and FTIR-spectroscopy, Fresenius' J. Anal. Chem. 338 (6) (1990) 710-716.

[26] F. Wang, H. Wu, Q. Zhu, K. Huang. Y. Wei, C. Liu, I. Noda, Development of narrow-band TLC plates for TLC/FTIR analysis, Anal. Methods 5 (16) (2013) 4138-4144.

[27] Y. Jiang, X. Kang, D. Gao, A. He, R. Guo, X. Fan, J. Wu, Finding a suitable separation condition for TLC/FTIR analysis by using multiple-narrow-band TLC technique, RSC Adv. 5 (28) (2015) 21544-21549.

[28] H. Yu, L. Liu, X. Wang, P. Wang, J. Yu, Y. Wang, The dependence of photocatalytic activity and photoinduced self-stability of photosensitive AgI nanoparticles, Dalton Trans. 41 (34) (2012) 10405-10411.

[29] P. Wang, B. Huang, Q. Zhang, X. Zhang, X. Qin, Y. Dai, Z. Lou, Highly efficient visible light plasmonic photocatalyst Ag@ $\mathrm{Ag}$ (Br, I), Chemistry-A Eur. J. 16 (33) (2010) 10042-10047.

[30] P. Wang, B. Huang, X. Qin, X. Zhang, Y. Dai, J. Wei, M.H. Whangbo, Ag@ AgCl: a highly efficient and stable photocatalyst active under visible light, Angew. Chem. Int. Ed. 47 (41) (2008) 7931-7933.

[31] B. Lei, M. Zhu, P. Chen, C. Chen, W. Ma, T. Li, M. Liu, Silver iodide microstructures of a uniform towerlike shape: morphology purification via a chemical dissolution, simultaneously boosted catalytic durability, and enhanced catalytic performances, ACS Appl. Mater. interfaces 6 (6) (2014) 4160-4169.

[32] R. Dong, B. Tian, C. Zeng, T. Li, T. Wang, J. Zhang, Ecofriendly synthesis and photocatalytic activity of uniform cubic Ag@ AgCl plasmonic photocatalyst, J. Phys. Chem. C 117 (1) (2012) 213-220.

[33] X. Wang, W. Shi, G. She, L. Mu, Surface-enhanced Raman scattering (SERS) on transition metal and semiconductor nanostructures, Phys. Chem. Chem. Phys. 14 (17) (2012) 5891-5901.

[34] L. Dawei, W. Jian, X. Houwen, S. Xu, L. Fan-chen, Enhancement origin of SERS from pyridine adsorbed on $\mathrm{AgCl}$ colloids, Spectrochim. Acta Part A Mol, Spectrosc. 43 (3) (1987) 379-382.

[35] H. Zhang, H. Xin, T. He, F. Liu, Time dependent surface enhanced Raman spectroscopy of pyridine in AgBr sol, Spectrochim. Acta Part A Mol. Spectrosc. 47 (7) (1991) 927-932.

[36] Q. Huang, S. Wen, X. Zhu, Synthesis and characterization of an AgI/Ag hybrid nanocomposite with surface-enhanced Raman scattering performance and photocatalytic activity, RSC Adv. 4 (70) (2014) 37187-37192.

[37] J.V. Goodpaster, E.A. Liszewski, Forensic analysis of dyed textile fibers, Anal. Bioanal. Chem. 394 (8) (2009).

[38] A. Idone, M. Gulmini, A.I. Henry, F. Casadio, L. Chang, L. Appolonia, N.C. Shah, Silver colloidal pastes for dye analysis of reference and historical textile fibers using direct, extraction less, non-hydrolysis surface-enhanced Raman spectroscopy, Analyst 138 (20) (2013) 5895-5903.

[39] A. Lehne, TabbelarischeUebersicht über diekünstlichen organsichen Farbstoffeund ihre Anwendung in Färberei und Zeugdrick, Verlag von Julius Springer, Berlin, 1893.

[40] X. Li, Y. Wang, H. Jia, W. Song, B. Zhao, An in situ reduction method for preparing novel surface-enhanced Raman scattering substrates, J. Raman Spectrosc. 36 (6-7) (2005) 635-639.

[41] W.A. Bassett, T. Takahashi, Silver iodide polymorphs, Am. Mineralogist 50 (10) (1965) 1576-1594.

[42] G. Burley, Polymorphism of silver iodide, Am. Mineralogist 48 (11-2) (1963) 1266.

[43] J.R.G. Patnaik, C.S. Sunandana, Studies on gamma silver iodide, J. Phys. Chem Solids 59 (6-7) (1998) 1059-1069.

[44] B.M. Weon, J.H. Je, Capillary force repels coffee-ring effect, Phys. Rev. E 82 (1) (2010) 015305.

[45] K. Kneipp, W. Jahr, G. Roewer, SERS on photographic silver halide emulsions, Chem. Phys. Lett. 163 (1) (1989) 105-110.

[46] Y. Han, Z. Liang, H. Sun, H. Xiao, H.L. Tsai, Nanostructured substrate with nanoparticles fabricated by femtosecond laser for surface-enhanced Raman scattering, Appl. Phys. A Mater. Sci. Process. 102 (2) (2011) 415-419.

[47] T. Baldacchini, A.C. Pons, J. Pons, C.N. LaFratta, J.T. Fourkas, Y. Sun, M.J. Naughton, Multiphoton laser direct writing of two-dimensional silver structures, Opt. express 13 (4) (2005) 1275-1280.

[48] A.L. Kamyshny, V.N. Zakharov, Y.V. Fedorov, A.E. Galashin, L.A. Aslanov, Surface-enhanced raman scattering of 2, $2^{\prime}$-bipyridine adsorbed on colloidal silver and stabilized AgBr sols, J. colloid interface Sci. 158 (1) (1993) 171-182. 